\title{
Being a doctor, being human
}

Renaissance humanism as a movement promotes returning to Greek-Roman culture as a means to restore human values, but this definition is not what comes to our minds when speaking of the human values that physicians should hold.

What is being asked of physicians when asked to be more human? Let us take a look at the definition provided by the Random House Learner's Dictionary of American English: "Human. adj. Of, relating to, characteristic of, or having the nature of people. || Sympathetic; kind. || A man, woman, or child."

As with many other things in life, it may also be defined by antagonism or the opposite. The antonym of human is cruel.

So we decided to conduct a survey among physicians to establish what "being human" meant to us. We also asked this question to $5^{\text {th }}$ and $6^{\text {th }}$ year students of the School of Medicine of Universidad Nacional de Córdoba. We wanted to find out what adjective was associated with the Human concept.

Ethical was the term most commonly suggested by students. Physicians, unlike students, found more relevance in the term Caring, which means "showing concern for others". This is probably because, throughout the course of medical practice, they have experienced this as a useful and tangible value, applicable to everyday activities.

Other outcome measures were also studied in both groups:

- Whether they had participated in any education activity (module, workshop, master class, etc.) on humanism.

- Those who answered YES were asked if they had considered it useful for their present or future professional development.

- Those who answered NO were asked if they believed that it would have helped them or if they considered that ethical and human principles learned from their families were enough.

Only half of students and physicians had participated in an education activity related to the humanities. This evidences that, from the perspective of curricular content and teaching approaches, this subject is considered insignificant. One hundred percent of students had taken Pharmacology, Microbiology or Anatomic Pathology, courses considered to be "serious", having ample resources and work load assigned to develop the proposed objectives. The humanities is sometimes only considered an activity, a break between "actually useful" courses.

However, when asked if the study of humanities would have been useful, all physicians answered affirmatively. This suggests that, in everyday practice with real people, physicians had required a stronger training in the humanities, and that the tools learned from their families were not enough, not because they were scarce but because they were now playing a different and difficult role with as many nuances as patients they had to see.

Communication skills, for example, are essential for developing empathy and a bond with patients so as to achieve a full and comprehensive understanding of their health/disease situation and context.

But this would be a partial view if we only considered the "doctor" component of the doctorthird-party (patient) dyad. So, in a different section of the same survey, we decided to ask patients what attributes they considered their physicians should have.

They were asked to rank the following attributes in order of importance:

A. Having good listening skills.

B. Being affectionate.

C. Having scientific knowledge.

D. Devoting more time to office visits.

Good listening skills came in first. These are critical for communication and the exchange of ideas. Technical and scientific capacity was pushed into second place. It seems that patients want humans, people to share their health / disease experience with and who also have medical knowledge. They look for a smart and skillful heart.

Medicine poses two poles of the same world: science or "scientism" (cold, abstract, logical) and humanism (strong, passionate, affectionate). From the balance between these two human medicine will be able to emerge. But these situations are larger than ourselves, they concern every professional activity. Lawyers and architects also fluctuate between these two stances. For this reason, it is essential for every man, whatever their profession or trade, to have a more human attitude.

To this end, it is necessary to cultivate human sciences, such as: 
- Words (language), as a communication experiment between two whole beings (body and soul).

- History, to provide a context to the reality and experiences of that "other being".

- Art, as a means to exalting the soul and values of men.

As stated by Mexican Professor Ruy Pérez Tamayo: "an educated physician is a better physician, not just because he/she is a physician but because he/she is a better human being".

In spite of standards, rules and works on "medical ethics", these usually refer to medicine and medical science rather than physicians as individuals. Professionals should be guided by "medical ethics", which should be the same as ordinary men's ethics. There are no dehumanized physicians; there are dehumanized people.

It is worth noting that the core of medicine is the doctor-patient relationship, a human relationship like many others, such as friendship or a father-child relationship, although its purpose is sometimes seeking health, and always bringing peace. As a human relationship, it requires an essential component: affection. We should add love to our medical practice; feelings bring people closer, overcome barriers, brighten things up, iron differences among people and their history.
In everyday medical and clinical practice, we may need better speaking and listening skills. Many times we will need to work from the heart, not just using our brains. We should awaken cosmic interests so that our light shines at everything. We should exercise medical and other type of knowledge through interdisciplinary and transdisciplinary activities in conjunction, each doing their part. We should learn to see life, people, and their reality, which are as varied as they are single and important, from a holistic perspective. Finally, we should also treasure a bit of a clown in our soul, so that tears and smiles live together in harmony, and keep hope and joy on edge, even in the most painful places of our soul.

Héctor Pedicino, M.D.

Pediatrician and Neonatologist. Assistant Head of the Department of Pediatrics and Neonatology,

Hospital Italiano de Córdoba. Professor of the Chair of Pediatrics, School of Medical Sciences, Universidad Nacional de Córdoba. Professor of the Chair of Medical Anthropology, School of Sciences, Universidad Nacional de Córdoba.

http:/ /dx.doi.org/10.5546/aap.2016.eng.502

\section{An attempt to include happiness within the psychiatric disorders}

\section{PREFACE}

The most recent edition of the Diagnostic and Statistical Manual of Mental Disorders (DSM-V) ${ }^{1}$ extends the range of new diagnostic criteria for adult and pediatric conditions. Since these criteria are so wide, there is a risk for a large portion of the general population to be included in one of the diseases described in the manual. On this basis, and in order to be consistent with the DSM-V, I thought it was appropriate to make a comment on an excellent article by Richard Bentall, from Liverpool University. ${ }^{2}$ His article is longer than this comment, and includes a more extensive bibliography, but here I describe the complexity of definitions and the classification of "disease" as a nosologic category established as necessarily true.

Doctor Bentall's considerations are quoted in italics.
ARTICLE DESCRIPTION
Bentall introduces his article by stating "Happiness is a phenomenon that has received very little attention from psychopathologists; for this reason, research on the topic of happiness has been rather limited. Nonetheless, the guidelines described 